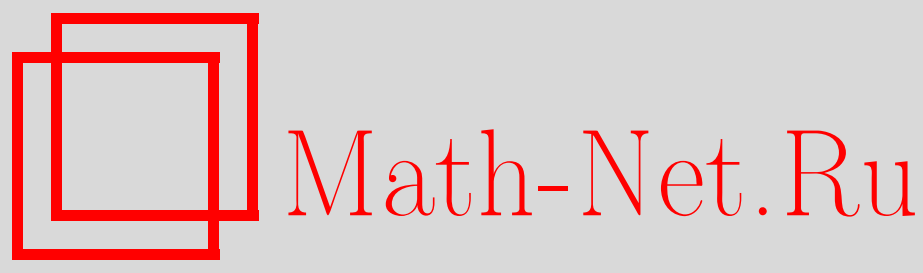

И. Т. Хабибуллин, А. Р. Хакимова, Инвариантные многообразия и пары Лакса для интегрируемых нелинейных цепочек, ТМФ, 2017, том 191, номер 3, 369-388

DOI: https://doi.org/10.4213/tmf9216

Использование Общероссийского математического портала Math-Net.Ru подразумевает, что вы прочитали и согласны с пользовательским соглашением http: //www . mathnet.ru/rus/agreement

Параметры загрузки:

IP : 35.174 .16 .151

26 апреля 2023 г., 11:51:25

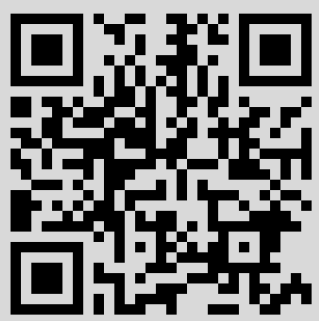




\title{
ФИЗИКА
}

Том 191, № 3

июнь, 2017

(C) 2017 г. $\quad$ И. Т. Хабибуллин*†, А. Р. Хакимова*†

\section{ИНВАРИАНТНЫЕ МНОГООБРАЗИЯ И ПАРЫ ЛАКСА ДЛЯ ИНТЕГРИРУЕМЫХ НЕЛИНЕЙНЫХ ЦЕПОЧЕК}

\begin{abstract}
Продолжены ранее начатые исследования по разработке прямого метода построения пары Лакса по заданному интегрируемому уравнению. В данном подходе не требуется никаких дополнительных предположений о свойствах уравнения. В качестве одного из уравнений пары Лакса взята линеаризация рассматриваемого нелинейного уравнения, а второе уравнение пары связано с его обобщенным инвариантным многообразием. Задача поиска второго уравнения сводится к простым, но довольно трудоемким вычислениям и, как показывают примеры, эффективно решается. Замечательный факт состоит в том, что второе уравнение пары позволяет легко отыскать рекурсионный оператор, описывающий иерархию высших симметрий уравнения. Полученные таким способом пары Лакса, на первый взгляд, отличаются от обычных, так как имеют более высокий порядок либо более высокую матричную размерность. На примерах показано, что понижением порядка они сводятся к обычным парам. В качестве примера рассматривается интегрируемая дважды дискретная система экспоненциального типа и ее высшая симметрия, для которой предъявлена пара Лакса и построены законы сохранения.
\end{abstract}

Ключевые слова: пара Лакса, интегрируемая цепочка, высшая симметрия, инвариантное многообразие, рекурсионный оператор.

DOI: https://doi.org/10.4213/tmf9216

\section{1. ВВЕДЕНИЕ}

В настоящей работе мы рассматриваем задачу о построении пары Лакса для нелинейного интегрируемого дифференциально-разностного уравнения (цепочки) вида

$$
\frac{d u_{n}}{d t}=f\left(u_{n+k}, u_{n+k-1}, \ldots, u_{n-k}\right)
$$

Исследование выполнено при финансовой поддержке гранта Российского научного фонда (проект № 15-11-20007).

* Институт математики с вычислительным центром Уфимского научного центра РАН, Уфа, Россия. E-mail: habibullinismagil@gmail.com, aigulya.khakimova@mail.ru

${ }^{\dagger}$ Башкирский государственный университет, Уфа, Россия 
где искомая функция $u=u_{n}(t)$ зависит от $t \in \mathbb{R}$ и $n \in \mathbb{Z}$. Хорошо известно, что интегрируемое уравнение, как правило, допускает представление Лакса, т. е. является условием совместности двух систем линейных уравнений, образующих пару Лакса. Представление Лакса - это эффективное средство изучения нелинейных уравнений, позволяющее находить интегралы движения, высшие симметрии, точные и асимптотические решения и т. д. В литературе можно найти различные подходы и методы поиска пар Лакса, от классических, таких как метод одевания по Захарову-Шабату [1], [2] и метод продолжения Уолквиста-Эстабрука [3], до более поздних, предложенных в работах [4]-[8]. В рамках этих подходов были найдены пары Лакса для очень важных классов интегрируемых моделей. Однако проблема эффективного алгоритма построения пары Лакса, пригодного для произвольного заданного интегрируемого уравнения, долгое время оставалась неизученной. Здесь уместно процитировать работу [9] (см. с. 1606): "Currently, there is not any general method for finding the Lax representation for a given equation". В нашей работе [10], написанной в соавторстве с Попцовой, был предложен новый подход к решению этой проблемы.

Для заданного интегрируемого уравнения в частных производных пара объектов, составленная из линеаризованного уравнения и уравнения инвариантного многообразия для линеаризованного уравнения, задает искомую пару Лакса. При этом, чтобы найти инвариантное многообразие, требуется выполнить сугубо вычислительную работу: решить переопределенную систему уравнений в частных производных. Многочисленные примеры убеждают, что эта переопределенная система эффективно решается.

В настоящей работе мы концентрируем внимание на дискретных цепочках. Следует отметить, что пары Лакса, полученные в [10] изложенным выше способом, являются настоящими, рабочими, парами, хотя имеют более высокий порядок или более высокую матричную размерность по сравнению с обычными парами Лакса. Поэтому мы называем их предварительными парами Лакса. В настоящей работе мы показываем на уровне примеров, что предварительные пары Лакса имеют первый интеграл и поэтому допускают понижение порядка, в результате чего они сводятся к некоторым нелинейным системам. Введением подходящих переменных полученные системы уравнений линеаризуются и преобразуются в обычные пары Лакса.

Кратко остановимся на содержании работы. В разделе 2 приводятся необходимые определения и излагается метод поиска предварительной $L-A$ пары. В разделе 3 иллюстрируется применение этого метода на примере известной интегрируемой нелинейной цепочки, которая является симметрией уравнения $\mathrm{dpKdV}$ (дискретного потенциального уравнения Кортевега-де Фриза). В разделе 4 обсуждается способ построения обобщенного инвариантного многообразия с помощью рекурсионного оператора. В разделе 5 рассматривается одна мало исследованная цепочка, связанная с цепочкой Вольтерра преобразованием типа Миуры. Прямым вычислением строится рекурсионный оператор для данной цепочки, с помощью которого вычисляется предварительная пара Лакса. Затем конструктивно ищется первый интеграл, позволяющий понизить порядок пары Лакса. Посредством формальной диагонализации пары Лакса построена бесконечная серия законов сохранения для рассматриваемой цепочки. В разделе 6 кратко обсуждается способ построения обобщенного инвариантного многообразия для дважды дискретного интегрируемого уравнения $\mathrm{dpKdV}$ с помощью его высшей симметрии. 


\section{2. ОБОБЩЕННОЕ ИНВАРИАНТНОЕ МНОГООБРАЗИЕ И ПАРА ЛАКСА НЕЛИНЕЙНОЙ ЦЕПОЧКИ}

При работе с уравнением (1) мы будем пользоваться стандартным набором динамических переменных $\left\{u_{j}\right\}_{j=-\infty}^{j=\infty}$. Напомним, что уравнение вида

$$
u_{n+s}=F\left(u_{n+s-1}, u_{n+s-2}, \ldots, u_{n}\right)
$$

определяет инвариантное многообразие для цепочки (1), если выполняется следующее условие:

$$
\frac{d}{d t} F-\left.D_{n}^{s} f\right|_{(1),(2)}=0
$$

где предполагается, что $d F / d u_{n}$ не равна нулю тождественно, а оператор сдвига $D_{n}$ действует по правилу $D_{n} u_{n}=u_{n+1}$. Применим к равенству (2) оператор $D_{n}^{-1}$ и запишем результат в виде, разрешенном относительно $u_{n-1}$ :

$$
u_{n-1}=G\left(u_{n+s-1}, u_{n+s-2}, \ldots, u_{n}\right) .
$$

Предположим, что функция $F$ неизвестна. Выразим в равенстве $(3)$ все производные $d u_{j} / d t$ с учетом уравнения (1), затем выразим все $u_{j}$ через переменные $u_{n+s-1}, u_{n+s-2}, \ldots, u_{n}$ с учетом уравнений $(2),(4)$ и их сдвигов. В результате получим некоторое, вообще говоря нелинейное, функционально-дифференциальное уравнение относительно искомой функции $F$. Это уравнение не является переопределенным, и поэтому оно, как правило, не решается в явном виде. Примеры инвариантных многообразий можно получить, взяв стационарные части высших симметрий.

Совсем другую картину мы имеем при работе с линеаризованным уравнением, т. е. уравнением, полученным из (1) посредством линеаризации в окрестности его произвольного решения $u=u_{n}(t)$ :

$$
\frac{d U_{n}}{d t}=\sum_{j=-k}^{k} A(j) U_{n+j},
$$

где $A(j)=\partial f / \partial u_{j}$.

ОПРЕДЕЛЕНиЕ. Будем называть обобщенным инвариантным многообразием уравнения (1) поверхность, заданную линейным уравнением

$$
U_{n+i}=\sum_{j=0}^{i-1} a(j) U_{n+j},
$$

если выполняется условие

$$
\frac{d}{d t} U_{n+i}-\left.D_{n}^{i} \frac{d U_{n}}{d t}\right|_{(1),(5),(6)}=0
$$

Предполагается, что функции $a(j)$ в уравнении (6) зависят от конечного числа динамических переменных $u_{j}$. Если в уравнении $(7)$ производные переменных $U_{j}$ и $u_{j}$ по $t$ заменить, учитывая уравнения (1) и (5), а значения переменной $U_{j}$ при 
$j<n$, а также при $j>n+i-1$ - с учетом уравнения (6), то получится равенство, которое должно выполняться тождественно по всем $u_{j},-\infty<j<+\infty$, а также тождественно по переменным $U_{j}$ для $n \leqslant j \leqslant n+i-1$. Сравнение коэффициентов при $U_{j}$ в полученном тождестве приводит к некоторой системе линейных дифференциальных уравнений для неизвестных $a(j)$ :

$$
\frac{d a(j)}{d t}=\sum_{s=0}^{i-1} b_{j, s} a(s), \quad j=\overline{0, i-1}
$$

где коэффициенты $b_{j, s}$ явно выражаются через коэффициенты $A(j)$ линеаризованного уравнения (5). Полученная система (8) является переопределенной, так как функции $a(j)$ должны зависеть только от конечного числа динамических переменных $u_{j}$. В силу переопределенности система (8) в принципе может быть решена в явном виде. Примеры можно найти в разделе 3 ниже, а также в работе [10].

Допустим, что коэффициенты уравнения (6) найдены. Тогда мы имеем систему уравнений (5), (6), которая совместна, если $u=u_{n}(t)$ является решением уравнения (1). Наша гипотеза состоит в том, что верно и обратное утверждение: для обобщенных инвариантных многообразий достаточно высокого порядка $i$ совместность системы (5), (6) влечет выполнение уравнения (1), т. е. система задает пару Лакса для уравнения (1).

\section{3. ПРИМЕР, ИЛЛЮСТРИРУЮЩИЙ ПРИМЕНЕНИЕ ПРЯМОГО АЛГОРИТМА ПОСТРОЕНИЯ ПАРЫ ЛАКСА}

Рассмотрим цепочку [11]

$$
\frac{d u_{n}}{d t}=\frac{1}{u_{n+1}-u_{n-1}}
$$

являющуюся симметрией уравнения $H_{1}$ из списка Адлера-Бобенко-Суриса [12]. Заметим, что коэффициенты линеаризованного уравнения

$$
\frac{d U_{n}}{d t}=-p_{n}^{2}\left(U_{n+1}-U_{n-1}\right)
$$

зависят только от $p_{n}=1 /\left(u_{n+1}-u_{n-1}\right)$. Легко проверить, что $p_{n}=p_{n}(t)$ является решением модифицированной цепочки Вольтерра

$$
\frac{d p}{d t}=-p_{n}^{2}\left(p_{n+1}-p_{n-1}\right)
$$

Обобщенное инвариантное многообразие, найденное в работе [10], имеет вид

$$
U_{n+2}=\left(-\frac{p_{n}}{p_{n+1}}+\frac{\lambda}{p_{n+1}^{2}}\right) U_{n+1}+\left(1-\frac{\lambda}{p_{n} p_{n+1}}\right) U_{n}+\frac{p_{n}}{p_{n+1}} U_{n-1} .
$$

Там же показано, что система (10), (12) задает пару Лакса для (11). Покажем, что путем понижения порядка систему (10), (12) можно свести к обычной паре Лакса. Для поиска “интеграла" уравнения (12) перепишем его в симметричном виде:

$$
p_{n+1} U_{n+2}-\frac{\lambda}{p_{n+1}} U_{n+1}-p_{n+1} U_{n}=-p_{n} U_{n+1}-\frac{\lambda}{p_{n}} U_{n}+p_{n} U_{n-1} .
$$


Возведем обе части этого равенства в квадрат и прибавим выражение $-4 \lambda U_{n} U_{n+1}$, тогда получим

$$
\begin{gathered}
p_{n+1}^{2} U_{n+2}^{2}+\frac{\lambda^{2}}{p_{n+1}^{2}} U_{n+1}^{2}+p_{n+1}^{2} U_{n}^{2}-2 \lambda U_{n+1} U_{n+2}-2 p_{n+1}^{2} U_{n} U_{n+2}-2 \lambda U_{n} U_{n+1}= \\
=p_{n}^{2} U_{n+1}^{2}+\frac{\lambda^{2}}{p_{n}^{2}} U_{n}^{2}+p_{n}^{2} U_{n-1}^{2}-2 \lambda U_{n} U_{n+1}-2 p_{n}^{2} U_{n-1} U_{n+1}-2 \lambda U_{n-1} U_{n} .
\end{gathered}
$$

Заметим, что последнее равенство имеет вид

$$
D_{n} I=I,
$$

где $I$ - искомый первый интеграл,

$$
I=p_{n}^{2} U_{n+1}^{2}+\frac{\lambda^{2}}{p_{n}^{2}} U_{n}^{2}+p_{n}^{2} U_{n-1}^{2}-2 \lambda U_{n} U_{n+1}-2 p_{n}^{2} U_{n-1} U_{n+1}-2 \lambda U_{n-1} U_{n} .
$$

Действительно, из (13) следует, что $I=C, C=$ const. Возьмем $C=0$, тогда уравнение (12) сведется к нелинейному уравнению второго порядка

$$
p_{n}^{2} U_{n+1}^{2}+\frac{\lambda^{2}}{p_{n}^{2}} U_{n}^{2}+p_{n}^{2} U_{n-1}^{2}+2 \lambda U_{n} U_{n+1}-2 p_{n}^{2} U_{n-1} U_{n+1}-2 \lambda U_{n-1} U_{n}=4 \lambda U_{n} U_{n+1},
$$

или

$$
\left(p_{n} U_{n+1}+\frac{\lambda}{p_{n}} U_{n}-p_{n} U_{n-1}\right)^{2}=4 \lambda U_{n} U_{n+1} .
$$

Извлечем квадратный корень из этого равенства:

$$
p_{n} U_{n+1}+\frac{\lambda}{p_{n}} U_{n}-p_{n} U_{n-1}=2 \varepsilon \sqrt{\lambda U_{n} U_{n+1}}, \quad \varepsilon= \pm 1 .
$$

Затем посредством замены $U_{n}=\varphi_{n}^{2}$ приведем уравнение к виду

$$
\left(\sqrt{p_{n}} \varphi_{n+1}-\varepsilon \frac{\sqrt{\lambda}}{\sqrt{p_{n}}} \varphi_{n}\right)^{2}=p_{n} \varphi_{n-1}^{2}
$$

В итоге приходим к линейному уравнению

$$
\varphi_{n+1}=\varepsilon \frac{\sqrt{\lambda}}{p_{n}} \varphi_{n}+\varphi_{n-1}
$$

Перепишем уравнение (10) эволюции по времени в новых переменных $\varphi_{n}$ :

$$
\frac{d}{d t} \varphi_{n}^{2}=-p_{n}^{2}\left(\varphi_{n+1}^{2}-\varphi_{n-1}^{2}\right)
$$

Заменим в последнем равенстве $\varphi_{n+1}$, учитывая уравнение $(14)$, и получим

$$
\varphi_{n, t}=-\frac{1}{2} \lambda \varphi_{n}-\varepsilon \sqrt{\lambda} p_{n} \varphi_{n-1}
$$

Легко видеть, что пара уравнений (14), (15) совпадает с известной парой Лакса для (11), которая связана с (9) линейной подстановкой $u_{n, t}=p_{n}$. 


\section{4. РЕКУРСИОННЫЙ ОПЕРАТОР И ОБОБЩЕННОЕ ИНВАРИАНТНОЕ МНОГООБРАЗИЕ}

Прямой алгоритм построения обобщенного инвариантного многообразия требует выполнения большого объема вычислений. Для систем нелинейных уравнений это становится особенно заметно. Работа существенно облегчается, если известен рекурсионный оператор уравнения (1). Тогда равенство

$$
R_{n} U_{n}=\lambda U_{n}, \quad \lambda \in \mathbb{C},
$$

также задает обобщенное инвариантное многообразие для уравнения (1). Поэтому система уравнений (5), (16) определяет пару Лакса для уравнения (1). Несмотря на то что рекурсионный оператор $R_{n}$ является нелокальным, введением дополнительных переменных можно свести систему (5), (16) к паре линейных систем дифференциальных и, соответственно, дискретных уравнений, которые задают пару Лакса для уравнения (1). С другой стороны, по известному обобщенному инвариантному многообразию достаточно высокого порядка для уравнения (1) легко можно построить его рекурсионный оператор.

Отметим, что полученная пара Лакса (5), (16) является предварительной парой Лакса, так как она имеет более высокий порядок, чем обычная пара. Дело в том, что решение $U_{n}$ уравнения (16) является "квадратом" собственной функции обычной пары Лакса. Ранее этот факт на примере уравнения синус-гордон отметил Сулейманов [13]. Связь пары Лакса с рекурсионным оператором была обнаружена еще в пионерских работах [14], [15].

\section{5. ПОСТРОЕНИЕ ПАРЫ ЛАКСА ДЛЯ ЦЕПОЧКИ, СВЯЗАННОЙ С ОДНОЙ АФФИННОЙ АЛГЕБРОЙ ЛИ СЕРИИ А}

Рассмотрим экспоненциальную систему, соответствующую алгебре Ли $A_{1}^{(1)}$ :

$$
\begin{aligned}
& e^{p_{n+1, m+1}}-e^{p_{n+1, m}}=e^{2 q_{n+1, m}-p_{n, m+1}-p_{n, m}}\left(e^{p_{n, m+1}}-e^{p_{n, m}}\right), \\
& e^{q_{n+1, m+1}}-e^{q_{n+1, m}}=e^{2 p_{n, m+1}-q_{n, m+1}-q_{n, m}}\left(e^{q_{n, m+1}}-e^{q_{n, m}}\right) .
\end{aligned}
$$

Эта система была найдена в работе [16]. Известно, что цепочки

$$
\begin{aligned}
p_{n, t}=e^{-p_{n, m}+q_{n+1, m}}+e^{p_{n, m}-q_{n, m}}, \quad q_{n, t}=e^{-p_{n-1, m}+q_{n, m}}+e^{p_{n, m}-q_{n, m}}, & \\
p_{n, \tau}= & e^{p_{n, m}-p_{n-1, m}}+2 e^{q_{n+1, m}-q_{n, m}}+e^{2\left(p_{n, m}-q_{n, m}\right)}+ \\
& +e^{p_{n+1, m}-p_{n, m}}+e^{2\left(q_{n+1, m}-p_{n, m}\right)}, \\
q_{n, \tau}= & e^{q_{n+1, m}-q_{n, m}}+2 e^{p_{n, m}-p_{n-1, m}}+e^{2\left(p_{n, m}-q_{n, m}\right)}+ \\
& +e^{q_{n, m}-q_{n-1, m}}+e^{2\left(q_{n, m}-p_{n-1, m}\right)},
\end{aligned}
$$

найденные в работе [16], являются симметриями системы (17). Точечной заменой переменных $e^{p_{n, m}}=u_{n, m}, e^{-q_{n, m}}=v_{n, m}$ приведем цепочки (18), (19) к удобному 
виду

$$
\begin{aligned}
& u_{n, t}=\frac{1}{v_{n+1}}+u_{n}^{2} v_{n}, \quad v_{n, t}=-\frac{1}{u_{n-1}}-u_{n} v_{n}^{2} \\
& u_{n, \tau}=\frac{u_{n}^{2}}{u_{n-1}}+2 \frac{u_{n} v_{n}}{v_{n+1}}+u_{n}^{3} v_{n}^{2}+u_{n+1}+\frac{1}{u_{n} v_{n+1}^{2}} \\
& v_{n, \tau}=\frac{v_{n}^{2}}{v_{n+1}}+2 \frac{u_{n} v_{n}}{u_{n-1}}+u_{n}^{2} v_{n}^{3}+v_{n-1}+\frac{1}{u_{n-1}^{2} v_{n}}
\end{aligned}
$$

Ниже для цепочки (20) будут построены рекурсионный оператор и пара Лакса. Насколько известно авторам, эти объекты для (20) ранее не были известны.

5.1. Построение рекурсионного оператора. Линеаризацию уравнения (20) можно записать в следующем виде:

$$
\frac{d}{d t}\left(\begin{array}{c}
U_{n} \\
V_{n}
\end{array}\right)=f_{n}^{*}\left(\begin{array}{c}
U_{n} \\
V_{n}
\end{array}\right)
$$

где оператор $f_{n}^{*}$, называемый оператором линеаризации, или производной Фреше, задается равенством

$$
f_{n}^{*}=F_{n}^{(1)} D_{n}+F_{n}^{(0)}+F_{n}^{(-1)} D_{n}^{-1} .
$$

Для коэффициентов оператора (22) имеем выражения

$$
F_{n}^{(1)}=\left(\begin{array}{cc}
0 & -\frac{1}{v_{n+1}^{2}} \\
0 & 0
\end{array}\right), \quad F_{n}^{(0)}=\left(\begin{array}{cc}
2 u_{n} v_{n} & u_{n}^{2} \\
-v_{n}^{2} & -2 u_{n} v_{n}
\end{array}\right), \quad F_{n}^{(-1)}=\left(\begin{array}{cc}
0 & 0 \\
\frac{1}{u_{n-1}^{2}} & 0
\end{array}\right) .
$$

Будем искать рекурсионный оператор в виде формального степенного ряда:

$$
L_{n}=\sum_{i=1}^{\infty} l_{n}^{(-i)} D_{n}^{-i}
$$

Ранее такой способ построения рекурсионного оператора для цепочек применялся в работе [17]. Отметим, что первым примером рекурсионного оператора в дискретном случае является оператор, построенный в [15] для цепочки Вольтерра. Подробное изложение теории рекурсионного оператора для цепочек и многочисленные примеры можно найти в работах [18], [9]. О рекурсионных операторах см. также работу [19].

Искомые коэффициенты ряда (23) вычисляются из уравнения

$$
\dot{L}_{n}=\left[f_{n}^{*}, L_{n}\right]
$$

где квадратные скобки обозначают коммутатор, оператор $\dot{L}_{n}$ получается из $L_{n}$ почленным дифференцированием по $t$ с использованием уравнений $(20)$. Имеет место следующее предложение.

ПреДЛОжениЕ. Решение $L_{n}$ уравнения (24) можно представить в виде суммы $L_{n}=L_{n}^{+}+L_{n}^{-}$, әде

$$
L_{n}^{+}=L_{n}^{(1)} D_{n}+L_{n}^{(0)}+L_{n}^{(-1)} D_{n}^{-1}, \quad L_{n}^{-}=\sum_{j=1}^{\infty} M_{n}^{(-j)} D_{n}^{-j} .
$$


Коэффициенты $L_{n}^{i}$ и $M_{n}^{(-j)}$ записъваются как

$$
\begin{gathered}
L_{n}^{(1)}=\left(\begin{array}{cc}
0 & -\frac{1}{v_{n+1}^{2}} \\
0 & 0
\end{array}\right), \quad L_{n}^{(0)}=\left(\begin{array}{cc}
2 u_{n} v_{n} & u_{n}^{2}-\frac{2 u_{n}}{u_{n-1} v_{n}^{2}} \\
-v_{n}^{2} & \frac{2}{u_{n-1} v_{n}}
\end{array}\right), \\
L_{n}^{(-1)}=\left(\begin{array}{cc}
0 & 0 \\
-\frac{1}{u_{n-1}^{2}} & 0
\end{array}\right), \\
M_{n}^{(-j)}=2\left(\begin{array}{r}
-u_{n} \\
v_{n}
\end{array}\right) D_{n}^{-j}\left(\frac{1}{u_{n}^{2} v_{n+1}}-v_{n}, \frac{1}{v_{n}^{2} u_{n-1}}-u_{n}\right), \quad j=\overline{1, \infty} .
\end{gathered}
$$

ДокАЗАТЕльство. Формулы (25) легко проверить непосредственной подстановкой, формулы (26) можно доказать по индукции.

СлеДСтвИЕ. Рекурсионный оператор имеет вид

$$
R_{n}=L_{n}^{+}+2\left(\begin{array}{r}
-u_{n} \\
v_{n}
\end{array}\right)\left(D_{n}-1\right)^{-1}\left(\frac{1}{u_{n}^{2} v_{n+1}}-v_{n}, \frac{1}{v_{n}^{2} u_{n-1}}-u_{n}\right) .
$$

Действительно, из (26) следует, что

$$
\begin{aligned}
L_{n}^{-} & =\sum_{j=1}^{\infty} M_{n}^{(-j)} D_{n}^{-j}= \\
& =2\left(\begin{array}{r}
-u_{n} \\
v_{n}
\end{array}\right)\left(D_{n}^{-1}+D_{n}^{-2}+\cdots+D_{n}^{-k}+\cdots\right)\left(\frac{1}{u_{n}^{2} v_{n+1}}-v_{n}, \frac{1}{v_{n}^{2} u_{n-1}}-u_{n}\right) .
\end{aligned}
$$

Имеем формальное равенство $D_{n}^{-1}+D_{n}^{-2}+\cdots+D_{n}^{-k}+\cdots=\left(D_{n}-1\right)^{-1}$, из которого получаем (27).

Непосредственной подстановкой можно убедиться, что оператор (27) переводит симметрию

$$
u_{n, \tau}=u_{n}, \quad v_{n, \tau}=-v_{n}
$$

цепочки (20) в саму цепочку, а также переводит цепочку (20) в ее высшую симметрию (21), т. е. оператор $R_{n}$ действительно является рекурсионным оператором для (20).

5.2. Обобщенное инвариантное многообразие. Выведем уравнение обобщенного инвариантного многообразия для (20) из уравнения (16), которое в данном случае принимает вид

$$
R_{n}\left(\begin{array}{c}
U_{n} \\
V_{n}
\end{array}\right)=\lambda\left(\begin{array}{c}
U_{n} \\
V_{n}
\end{array}\right)
$$

Из этого уравнения имеем

$$
\left(L_{n}^{(1)} D_{n}+L_{n}^{(0)}+L_{n}^{(-1)} D_{n}^{-1}\right)\left(\begin{array}{c}
U_{n} \\
V_{n}
\end{array}\right)+2\left(\begin{array}{r}
-u_{n} \\
v_{n}
\end{array}\right) W_{n}=\lambda\left(\begin{array}{c}
U_{n} \\
V_{n}
\end{array}\right),
$$

где

$$
W_{n}=\left(D_{n}-1\right)^{-1}\left(\left(\frac{1}{u_{n}^{2} v_{n+1}}-v_{n}\right) U_{n}+\left(\frac{1}{v_{n}^{2} u_{n-1}}-u_{n}\right) V_{n}\right) .
$$


Перепишем (28) в развернутом виде:

$$
\begin{aligned}
& -\frac{1}{v_{n+1}^{2}} V_{n+1}+2 u_{n} v_{n} U_{n}+\left(u_{n}^{2}-\frac{2 u_{n}}{u_{n-1} v_{n}^{2}}\right) V_{n}-2 u_{n} W_{n}-\lambda U_{n}=0, \\
& -v_{n}^{2} U_{n}+\frac{2}{u_{n-1} v_{n}} V_{n}-\frac{1}{u_{n-1}^{2}} U_{n-1}+2 v_{n} W_{n}-\lambda V_{n}=0 .
\end{aligned}
$$

Из (29) получаем уравнение

$$
W_{n+1}=\left(\frac{1}{u_{n}^{2} v_{n+1}}-v_{n}\right) U_{n}+\left(\frac{1}{v_{n}^{2} u_{n-1}}-u_{n}\right) V_{n}+W_{n} .
$$

Подействуем оператором сдвига $D_{n}$ на второе уравнение системы $(30)$ и заменим в полученном равенстве $W_{n+1}$ на основе $(31)$. В результате получим соотношения, связывающие переменные $U_{n}, V_{n}, U_{n+1}, V_{n+1}, W_{n}$ :

$$
\begin{aligned}
-\frac{1}{v_{n+1}^{2}} V_{n+1} & +2 u_{n} v_{n} U_{n}+\left(u_{n}^{2}-\frac{2 u_{n}}{u_{n-1} v_{n}^{2}}\right) V_{n}-2 u_{n} W_{n}-\lambda U_{n}=0 \\
-v_{n+1}^{2} U_{n+1} & +\frac{2}{u_{n} v_{n+1}} V_{n+1}-\frac{1}{u_{n}^{2}} U_{n}+2 v_{n+1}\left(\frac{1}{u_{n}^{2} v_{n+1}}-v_{n}\right) U_{n}+ \\
& +2 v_{n+1}\left(\frac{1}{v_{n}^{2} u_{n-1}}-u_{n}\right) V_{n}+2 v_{n+1} W_{n}-\lambda V_{n+1}=0 .
\end{aligned}
$$

Выразим из системы (32) переменные $U_{n+1}, V_{n+1}$. Тогда с учетом (31) получим дискретную систему

$$
\begin{aligned}
U_{n+1}= & \frac{\left(2 u_{n}^{2} v_{n} v_{n+1}-\lambda u_{n} v_{n+1}+1\right)\left(1-\lambda u_{n} v_{n+1}\right)}{u_{n}^{2} v_{n+1}^{2}} U_{n}- \\
& -\frac{2+\lambda u_{n}^{2} u_{n-1} v_{n}^{2} v_{n+1}-2 \lambda u_{n} v_{n+1}}{v_{n}^{2} u_{n-1} v_{n+1}} V_{n}+\frac{2\left(\lambda u_{n} v_{n+1}-1\right)}{v_{n+1}} W_{n}, \\
V_{n+1}= & v_{n+1}^{2}\left(2 u_{n} v_{n}-\lambda\right) U_{n}+\frac{u_{n} v_{n+1}^{2}\left(u_{n-1} u_{n} v_{n}^{2}-2\right)}{u_{n-1} v_{n}^{2}} V_{n}-2 u_{n} v_{n+1}^{2} W_{n}, \\
W_{n+1}= & \left(\frac{1}{u_{n}^{2} v_{n+1}}-v_{n}\right) U_{n}+\left(\frac{1}{v_{n}^{2} u_{n-1}}-u_{n}\right) V_{n}+W_{n} .
\end{aligned}
$$

Доопределим действие оператора $d / d t$ на переменную $W_{n}$. Для этого продифференцируем уравнение (29) и воспользуемся равенством

$$
\begin{aligned}
& \frac{d}{d t}\left(\left(\frac{1}{u_{n}^{2} v_{n+1}}-v_{n}\right) U_{n}+\left(\frac{1}{v_{n}^{2} u_{n-1}}-u_{n}\right) V_{n}\right)= \\
& \quad=\left(D_{n}-1\right)\left(\frac{u_{n} u_{n-1} v_{n}^{2}-1}{u_{n-1}^{3} v_{n}^{2}} U_{n-1}+\frac{u_{n-1}^{2} v_{n-1} v_{n}-1}{u_{n-1}^{2} v_{n}^{3}} V_{n}\right),
\end{aligned}
$$

являющимся законом сохранения линеаризованного уравнения (22). В итоге получим уравнение

$$
W_{n, t}=\frac{u_{n} u_{n-1} v_{n}^{2}-1}{u_{n-1}^{3} v_{n}^{2}} U_{n-1}+\frac{u_{n-1}^{2} v_{n-1} v_{n}-1}{u_{n-1}^{2} v_{n}^{3}} V_{n} .
$$


Исключим $U_{n-1}$, используя уравнение $(30)$, и найдем, что эволюция по $t$ переменных $U_{n}, V_{n}, W_{n}$ задается системой

$$
\begin{aligned}
U_{n, t}= & \lambda U_{n}+\frac{2 u_{n}}{u_{n-1} v_{n}^{2}} V_{n}+2 u_{n} W_{n} \\
V_{n, t}= & -2 v_{n}^{2} U_{n}-\left(\frac{2 u_{n} u_{n-1} v_{n}^{2}+\lambda u_{n-1} v_{n}-2}{v_{n} u_{n-1}}\right) V_{n}+2 v_{n} W_{n} \\
W_{n, t}= & \frac{1-u_{n-1} u_{n} v_{n}^{2}}{u_{n-1}} U_{n}-\left(\frac{3}{u_{n-1}^{2} v_{n}^{3}}-\frac{\lambda-u_{n} u_{n-1} v_{n}^{2} \lambda+2 u_{n} v_{n}+u_{n-1} v_{n-1}}{u_{n-1} v_{n}^{2}}\right) V_{n}- \\
& -\frac{2\left(1-u_{n-1} u_{n} v_{n}^{2}\right)}{u_{n-1} v_{n}} W_{n} .
\end{aligned}
$$

Непосредственным вычислением можно проверить, что система уравнений (33), (34) задает пару Лакса для (20).

5.3. Первый интеграл. Пример, рассмотренный в разделе 3 , показывает, что предварительная пара Лакса, вычисленная при помощи линейного инвариантного многообразия, допускает первый интеграл, который позволяет понизить ее порядок. Нам удобно вместо самого́ первого интеграла найти сначала равносильное ему условие связи

$$
U_{n}=H_{n}\left(V_{n}, W_{n}, u_{n-1}, v_{n-1}, u_{n}, v_{n}, u_{n+1}, v_{n+1}\right),
$$

совместное с уравнениями (33), (34). Следует отметить, что здесь мы ищем интеграл не для одной отдельно взятой системы, а для целого семейства систем уравнений, зависящих от динамических переменных $u_{n}, v_{n}$ и их сдвигов. Поэтому искомый интеграл должен также зависеть от этих переменных. В результате задача поиска интеграла становится переопределенной и успешно решается.

Подставим (35) в первое уравнение системы (34). Все производные переменных $V_{n}, W_{n}, u_{n-1}, v_{n-1}, u_{n}, v_{n}, u_{n+1}, v_{n+1}$ по переменной $t$ заменим, учитывая уравнения (34) и (20). Тогда получим

$$
\begin{aligned}
\lambda H_{n} & +\frac{2 u_{n}}{u_{n-1} v_{n}^{2}} V_{n}+2 u_{n} W_{n}+ \\
& +\left(2 v_{n}^{2} H_{n}+\frac{2 u_{n-1} u_{n} v_{n}^{2}+\lambda u_{n-1} v_{n}-2}{u_{n-1} v_{n}} V_{n}-2 v_{n} W_{n}\right) H_{n, V_{n}}+ \\
& +\left(\frac{u_{n-1} u_{n} v_{n}^{2}-1}{u_{n-1}} H_{n}-\frac{2\left(u_{n-1} u_{n} v_{n}^{2}-1\right)}{u_{n-1} v_{n}} W_{n}+\right. \\
& \left.+\frac{3-\lambda u_{n-1} v_{n}-2 u_{n-1} u_{n} v_{n}^{2}+\lambda u_{n-1}^{2} u_{n} v_{n}^{3}-u_{n-1}^{2} v_{n-1} v_{n}}{u_{n-1}^{2} v_{n}^{3}} V_{n}\right) H_{n, W_{n}}- \\
& -\left(\frac{1}{v_{n}}+u_{n-1}^{2} v_{n-1}\right) H_{n, u_{n-1}}+\left(\frac{1}{u_{n-2}}+u_{n-1} v_{n-1}^{2}\right) H_{n, v_{n-1}}- \\
& -\left(\frac{1}{v_{n+1}}+u_{n}^{2} v_{n}\right) H_{n, u_{n}}+\left(\frac{1}{u_{n-1}}+u_{n} v_{n}^{2}\right) H_{n, v_{n}}- \\
& -\left(\frac{1}{v_{n+2}}+u_{n+1}^{2} v_{n+1}\right) H_{n, u_{n+1}}+\left(\frac{1}{u_{n}}+u_{n+1} v_{n+1}^{2}\right) H_{n, v_{n+1}}=0 .
\end{aligned}
$$


Здесь $H_{n, V_{n}}:=\partial H_{n} / \partial V_{n}$ и т.д. Сравнивая в равенстве (36) коэффициенты при $1 / u_{n-2}, 1 / v_{n+2}$, получаем $H_{n, v_{n-1}}=H_{n, u_{n+1}}=0$. Следовательно, функция $H_{n}$ не зависит от переменных $v_{n-1}$ и $u_{n+1}$, т. е. $H_{n}=H_{n}\left(V_{n}, W_{n}, u_{n-1}, u_{n}, v_{n}, v_{n+1}\right)$. Отсюда, сравнивая в (36) коэффициенты при $u_{n+1}$, находим уравнение

$$
H_{n, v_{n+1}}=0 \quad \Longrightarrow \quad H_{n}=H_{n}\left(V_{n}, W_{n}, u_{n-1}, u_{n}, v_{n}\right)
$$

Подставим его в $(36)$ и приравняем коэффициенты при $1 / v_{n+1}$, получим

$$
H_{n, u_{n}}=0 \quad \Longrightarrow \quad H_{n}=H_{n}\left(V_{n}, W_{n}, u_{n-1}, v_{n}\right)
$$

В результате этих уточнений уравнение (36) принимает вид

$$
\begin{aligned}
\lambda H_{n}+ & \frac{2 u_{n}}{u_{n-1} v_{n}^{2}} V_{n}+2 u_{n} W_{n}+ \\
& +\left(2 v_{n}^{2} H_{n}+\frac{2 u_{n-1} u_{n} v_{n}^{2}+\lambda u_{n-1} v_{n}-2}{u_{n-1} v_{n}} V_{n}-2 v_{n} W_{n}\right) H_{n, V_{n}}+ \\
& +\left(\frac{u_{n-1} u_{n} v_{n}^{2}-1}{u_{n-1}} H_{n}-\frac{2\left(u_{n-1} u_{n} v_{n}^{2}-1\right)}{u_{n-1} v_{n}} W_{n}+\right. \\
& \left.\quad+\frac{3-\lambda u_{n-1} v_{n}-2 u_{n-1} u_{n} v_{n}^{2}+\lambda u_{n-1}^{2} u_{n} v_{n}^{3}-u_{n-1}^{2} v_{n-1} v_{n}}{u_{n-1}^{2} v_{n}^{3}} V_{n}\right) H_{n, W_{n}}- \\
& -\left(\frac{1}{v_{n}}+u_{n-1}^{2} v_{n-1}\right) H_{n, u_{n-1}}+\left(\frac{1}{u_{n-1}}+u_{n} v_{n}^{2}\right) H_{n, v_{n}}=0 .
\end{aligned}
$$

В равенстве (37) приравняем коэффициенты при $v_{n-1}$ :

$$
-\frac{1}{v_{n}^{2}} V_{n} H_{n, W_{n}}-u_{n-1}^{2} H_{n, u_{n-1}}=0 .
$$

Отсюда следует, что

$$
H_{n}=H_{n}\left(V_{n}, v_{n}, \theta_{n}\right), \quad \text { где } \quad \theta_{n}=\frac{u_{n-1} v_{n}^{2} W_{n}+V_{n}}{u_{n-1} V_{n}} .
$$

Перепишем (37) в новых переменных:

$$
\begin{aligned}
\frac{2 u_{n} \theta_{n}}{v_{n}^{2}} V_{n} & +\lambda H_{n}+\left(2 u_{n} v_{n} V_{n}-\frac{2 \theta_{n}}{v_{n}} V_{n}+2 v_{n}^{2} H_{n}+\lambda V_{n}\right) H_{n, V_{n}}+ \\
& +\left(\frac{1}{u_{n-1}}+u_{n} v_{n}^{2}\right) H_{n, v_{n}}+ \\
& +\left(\frac{v_{n}^{2}}{u_{n-1} V_{n}} H_{n}+\lambda u_{n} v_{n}^{2}-\frac{2 v_{n}^{2} \theta_{n}}{V_{n}} H_{n}-\lambda \theta_{n}-2 u_{n} v_{n} \theta_{n}+\right. \\
& \left.+\frac{2 \theta_{n}^{2}}{v_{n}}+\frac{2 \theta_{n}}{u_{n-1} v_{n}}+\frac{u_{n} v_{n}^{4}}{V_{n}} H_{n}\right) H_{n, \theta_{n}}=0
\end{aligned}
$$


Сравнение коэффициентов при независимых переменных $u_{n-1}, u_{n}$ показывает, что уравнение (38) равносильно следующей системе трех уравнений:

$$
\begin{gathered}
H_{n, v_{n}}+\left(\frac{v_{n}^{2}}{V_{n}} H_{n}+\frac{2 \theta_{n}}{v_{n}}\right) H_{n, \theta_{n}}=0, \\
2 v_{n} V_{n} H_{n, V_{n}}+v_{n}^{2} H_{n, v_{n}}+\frac{v_{n}\left(\lambda v_{n} V_{n}-2 \theta_{n} V_{n}+v_{n}^{3} H_{n}\right)}{V_{n}} H_{n, \theta_{n}}+\frac{2 \theta_{n}}{v_{n}^{2}} V_{n}=0, \\
\frac{2 v_{n}^{3} H_{n}-2 \theta_{n} V_{n}+\lambda v_{n} V_{n}}{v_{n}} H_{n, V_{n}}+\lambda H_{n}-\frac{\theta_{n}\left(2 v_{n}^{3} H_{n}-2 \theta_{n} V_{n}+\lambda v_{n} V_{n}\right)}{v_{n} V_{n}} H_{n, \theta_{n}}=0 .
\end{gathered}
$$

Из уравнения (40) вычтем уравнение (39), умноженное на $v_{n}^{2}$ :

$$
2 v_{n} V_{n} H_{n, V_{n}}+\frac{2 \theta_{n}}{v_{n}^{2}} V_{n}+\left(\lambda v_{n}-4 \theta_{n}\right) v_{n} H_{n, \theta_{n}}=0
$$

Далее умножим уравнение (41) на $v_{n}$ и вычтем из него уравнение (42), умноженное на $\frac{2 v_{n}^{3} H_{n}+\lambda v_{n} V_{n}-2 \theta_{n} V_{n}}{2 v_{n} V_{n}}$, тогда получим

$$
\frac{1}{2} \frac{2 v_{n}^{3} H_{n}-2 \theta_{n} V_{n}+\lambda v_{n} V_{n}}{V_{n}} H_{n, \theta_{n}}-\frac{v_{n}^{3} H_{n}-\theta_{n} V_{n}}{v_{n}^{3}}=0 .
$$

Решение этого уравнения имеет вид

$$
H_{n}\left(V_{n}, v_{n}, \theta_{n}\right)=\frac{1}{v_{n}^{3}}\left(\theta_{n} V_{n}-\frac{1}{2} \lambda v_{n} V_{n}-\frac{1}{2} V_{n} \sqrt{-4 \lambda \theta_{n} v_{n}+\lambda^{2} v_{n}^{2}+4 \lambda v_{n} F\left(V_{n}, v_{n}\right)}\right)
$$

где $F\left(V_{n}, v_{n}\right)$ - неизвестная функция. Найдем ее из оставшихся уравнений. Подставим (43) в (39) и получим

$$
F\left(V_{n}, v_{n}\right)=f\left(V_{n}\right) v_{n}^{3}
$$

Далее из (40) находим, что

$$
f\left(V_{n}\right)=\frac{c}{V_{n}^{2}},
$$

где $c$ - постоянная. Следовательно, первый интеграл имеет вид

$$
U_{n}=-\frac{1}{2 v_{n}^{3}}\left(-2 \theta_{n} V_{n}+\lambda v_{n} V_{n}+\sqrt{\lambda v_{n}\left(-4 \theta_{n} V_{n}^{2}+\lambda v_{n} V_{n}^{2}+4 c v_{n}^{3}\right)}\right) .
$$

Подставим $\theta_{n}=\frac{u_{n-1} v_{n}^{2} W_{n}+V_{n}}{u_{n-1} V_{n}}$ в (44) и получим равенство

$$
\begin{aligned}
U_{n}= & -\frac{1}{2 v_{n}^{3}}\left(-\frac{2\left(u_{n-1} v_{n}^{2} W_{n}+V_{n}\right)}{u_{n-1}}+\lambda v_{n} V_{n}+\right. \\
& \left.+\sqrt{\frac{\lambda v_{n}}{u_{n-1}}\left(-4 u_{n-1} v_{n}^{2} V_{n} W_{n}-4 V_{n}^{2}+\lambda u_{n-1} v_{n} V_{n}^{2}+4 c u_{n-1} v_{n}^{3}\right)}\right),
\end{aligned}
$$

которое задает искомый первый интеграл (точнее, условие связи (35)) для систем уравнений (33), (34). 
5.4. Понижение порядка. Введением новой переменной $P_{n}=v_{n}^{2} W_{n}+V_{n} / u_{n-1}$ сведем уравнения (33), (34) к следующему виду:

$$
\begin{aligned}
U_{n+1}= & \frac{\left(2 u_{n}^{2} v_{n} v_{n+1}-\lambda u_{n} v_{n+1}+1\right)\left(1-\lambda u_{n} v_{n+1}\right)}{u_{n}^{2} v_{n+1}^{2}} U_{n}- \\
& -\lambda u_{n}^{2} V_{n}+\frac{2\left(\lambda u_{n} v_{n+1}-1\right)}{v_{n}^{2} v_{n+1}} P_{n}, \\
V_{n+1}= & v_{n+1}^{2}\left(2 u_{n} v_{n}-\lambda\right) U_{n}+u_{n}^{2} v_{n+1}^{2} V_{n}-\frac{2 u_{n} v_{n+1}^{2}}{v_{n}^{2}} P_{n}, \\
P_{n+1}= & \frac{v_{n+1}\left(u_{n}^{2} v_{n} v_{n+1}-\lambda u_{n} v_{n+1}+1\right)}{u_{n}^{2}} U_{n}-\frac{v_{n+1}^{2}}{v_{n}^{2}} P_{n} . \\
U_{n, t}= & \lambda U_{n}+\frac{2 u_{n}}{v_{n}^{2}} P_{n}, \\
V_{n, t}= & -2 v_{n}^{2} U_{n}-\left(\lambda+2 u_{n} v_{n}\right) V_{n}+\frac{2}{v_{n}} P_{n}, \\
P_{n, t}= & -\frac{v_{n}^{2}\left(1+u_{n-1} u_{n} v_{n}^{2}\right)}{u_{n-1}} U_{n}-\lambda u_{n} v_{n}^{2} V_{n}-\frac{2}{u_{n-1} v_{n}} P_{n} .
\end{aligned}
$$

Первый интеграл (45) также перепишем в новых переменных. Положим $c=0$, в результате имеем

$$
U_{n}=-\frac{1}{2 v_{n}^{3}}\left(-2 P_{n}+\lambda v_{n} V_{n}+\sqrt{\lambda v_{n} V_{n}\left(-4 P_{n}+\lambda v_{n} V_{n}\right)}\right) .
$$

Введем новые переменные $\varphi_{n}, \psi_{n}$ так, чтобы из этого равенства извлекался корень. Для этого положим

$$
V_{n}=\frac{1}{\lambda v_{n}} \varphi_{n}^{2}, \quad P_{n}=\frac{1}{4}\left(\varphi_{n}^{2}-\psi_{n}^{2}\right)
$$

тогда

$$
U_{n}=-\frac{1}{4 v_{n}^{3}}\left(\varphi_{n}+\psi_{n}\right)^{2}
$$

В новых переменных система (46) записывается как

$$
\begin{aligned}
\varphi_{n+1} & =-\frac{1}{2} \frac{v_{n+1}^{3 / 2}}{v_{n}^{3 / 2}}\left(\lambda-2 u_{n} v_{n}\right) \varphi_{n}-\frac{1}{2} \frac{v_{n+1}^{3 / 2}}{v_{n}^{3 / 2}} \lambda \psi_{n} \\
\psi_{n+1} & =\frac{1}{2} \frac{v_{n+1}^{1 / 2}}{u_{n} v_{n}^{3 / 2}}\left(2 u_{n}^{2} v_{n} v_{n+1}-\lambda u_{n} v_{n+1}+2\right) \varphi_{n}+\frac{1}{2} \frac{v_{n+1}^{1 / 2}}{u_{n} v_{n}^{3 / 2}}\left(2-\lambda u_{n} v_{n+1}\right) \psi_{n} .
\end{aligned}
$$

Система (47) приводится к виду

$$
\begin{aligned}
& \varphi_{n, t}=-\frac{1}{2} \frac{\left(1+3 u_{n-1} u_{n} v_{n}^{2}\right)}{u_{n-1} v_{n}} \varphi_{n}+\frac{1}{2} \lambda \psi_{n}, \\
& \psi_{n, t}=-\frac{1}{2} \frac{\left(2 u_{n-1} u_{n} v_{n}^{2}-\lambda u_{n-1} v_{n}+2\right)}{u_{n-1} v_{n}} \varphi_{n}-\frac{1}{2} \frac{\left(3+u_{n-1} u_{n} v_{n}^{2}\right)}{u_{n-1} v_{n}} \psi_{n} .
\end{aligned}
$$


Для упрощения сделаем замену $\varphi_{n}=v_{n}^{3 / 2} \tilde{\varphi}_{n}, \psi_{n}=v_{n}^{3 / 2} \tilde{\psi}_{n}$ и найдем окончательную форму пары Лакса для (20):

$$
\begin{aligned}
\tilde{\varphi}_{n+1} & =\left(u_{n} v_{n}-\xi\right) \tilde{\varphi}_{n}-\xi \tilde{\psi}_{n} \\
\tilde{\psi}_{n+1} & =\left(u_{n} v_{n}+\frac{1}{u_{n} v_{n+1}}-\xi\right) \tilde{\varphi}_{n}+\left(\frac{1}{u_{n} v_{n+1}}-\xi\right) \tilde{\psi}_{n} \\
\tilde{\varphi}_{n, t} & =\frac{1}{u_{n-1} v_{n}} \tilde{\varphi}_{n}+\xi \tilde{\psi}_{n} \\
\tilde{\psi}_{n, t} & =-\left(u_{n} v_{n}+\frac{1}{u_{n-1} v_{n}}-\xi\right) \tilde{\varphi}_{n}+u_{n} v_{n} \tilde{\psi}_{n}
\end{aligned}
$$

где $\xi=\lambda / 2$.

5.5. Построение законов сохранения с помощью пары Лакса. Построим методом формальной диагонализации пары Лакса (см. работы [20]-[22]) законы сохранения для цепочки (20). Положим $\widetilde{\Phi}_{n}=\left(\tilde{\varphi}_{n}, \tilde{\psi}_{n}\right)^{\mathrm{T}}, Y_{n}=\left(y_{n}, z_{n}\right)^{\mathrm{T}}$.

Линейным преобразованием $\widetilde{\Phi}_{n}=\widetilde{T} Y_{n}$, где

$$
\widetilde{T}=\left(\begin{array}{rr}
1 & 1 \\
1 & -1
\end{array}\right)
$$

приведем систему уравнений (48) к виду

$$
Y_{n, t}=(a \xi+Q) Y_{n}, \quad Y_{n+1}=g Y_{n}
$$

где $a=\operatorname{diag}(1,-1)$ - диагональная матрица, а коэффициенты $Q, g$ имеют вид

$$
Q=\left(\begin{array}{cc}
0 & -u_{n} v_{n} \\
\frac{1}{u_{n-1} v_{n}} & \frac{1}{u_{n-1} v_{n}}+u_{n} v_{n}
\end{array}\right), \quad g=\left(\begin{array}{cc}
-2 \xi+u_{n} v_{n}+\frac{1}{u_{n} v_{n+1}} & u_{n} v_{n} \\
-\frac{1}{u_{n} v_{n+1}} & 0
\end{array}\right) .
$$

Формальной заменой переменных $Y_{n}=T^{-1} \Psi_{n}$ приведем систему (49), (50) к диагональному виду (см. работу [20])

$$
\Psi_{n, t}=(a \xi+h) \Psi_{n}, \quad \Psi_{n+1}=S \Psi_{n} .
$$

Здесь $T, h$ и $S$ - формальные ряды по степеням $\xi$,

$$
\begin{aligned}
& T=\left(\begin{array}{ll}
1 & 0 \\
0 & 1
\end{array}\right)+\left(\begin{array}{cc}
0 & -\frac{1}{2} u_{n} v_{n} \\
-\frac{1}{2 u_{n-1} v_{n}} & 0
\end{array}\right) \xi^{-1}+\left(\begin{array}{cc}
0 & -\frac{v_{n}+u_{n}^{2} v_{n}^{2} v_{n+1}}{4 v_{n+1}} \\
-\frac{1+u_{n-1}^{2} v_{n-1} v_{n}}{4 u_{n-1}^{2} v_{n}^{2}} & 0
\end{array}\right) \xi^{-2}+\cdots, \\
& h=\left(\begin{array}{cc}
0 & 0 \\
0 & -\frac{1+u_{n-1} u_{n} v_{n}^{2}}{u_{n-1} v_{n}}
\end{array}\right)+\left(\begin{array}{cc}
\frac{u_{n}}{2 u_{n-1}} & 0 \\
0 & -\frac{u_{n}}{2 u_{n-1}}
\end{array}\right) \xi^{-1}+\left(\begin{array}{cc}
\frac{1+u_{n}^{2} v_{n} v_{n+1}}{4 u_{n-1} v_{n+1}} & 0 \\
0 & -\frac{u_{n}\left(1+u_{n-1}^{2} v_{n} v_{n-1}\right)}{4 u_{n-1}^{2} v_{n}}
\end{array}\right) \xi^{-2}+ \\
& +\left(\begin{array}{cc}
\frac{u_{n}^{4} v_{n}^{2} v_{n+1}^{2}+2 u_{n}^{2} v_{n} v_{n+1}+u_{n+1} v_{n+1}^{2} u_{n}+1}{8 u_{n} u_{n-1} v_{n+1}^{2}} & -\frac{u_{n}\left(1+2 u_{n-1}^{2} v_{n-1} v_{n}\right)}{8 u_{n-1}^{3} v_{n}^{2}}-\frac{u_{n}\left(1+u_{n-1} v_{n-1}^{2} u_{n-2}\right)}{8 u_{n-2}} \\
0 & -\frac{u^{-3}+\cdots,}{0}
\end{array}\right.
\end{aligned}
$$




$$
\begin{aligned}
S=\left(\begin{array}{rr}
-2 & 0 \\
0 & 0
\end{array}\right) \xi+\left(\begin{array}{cc}
u_{n} v_{n}+\frac{1}{u_{n} v_{n+1}} & 0 \\
0 & 0
\end{array}\right)+\left(\begin{array}{cc}
\frac{u_{n+1}}{2 u_{n}} & 0 \\
0 & -\frac{v_{n}}{2 v_{n+1}}
\end{array}\right) \xi^{-1}+ \\
+\left(\begin{array}{cc}
\frac{1+u_{n+1}^{2} v_{n+1} v_{n+2}}{4 u_{n} v_{n+2}} & 0 \\
0 & -\frac{v_{n}\left(1+u_{n}^{2} v_{n} v_{n+1}\right)}{4 u_{n} v_{n+1}^{2}}
\end{array}\right) \xi^{-2}+\cdots
\end{aligned}
$$

Отметим, что все коэффициенты рядов для $h$ и $S$ являются диагональными матрицами, а все коэффициенты ряда для $T$, кроме первого, имеют нулевую диагональ. Коэффициенты рядов $T$ и $h$ вычисляются рекуррентно из цепочки уравнений

$$
\begin{aligned}
-E a & =-a E, \\
E \frac{d}{d t}-E Q-T_{1} a & =\frac{d}{d t} E+h_{0} E-a T_{1}, \\
T_{1} \frac{d}{d t}-T_{1} Q-T_{2} a & =\frac{d}{d t} T_{1}+h_{0} T_{1}+h_{1} E-a T_{2}, \\
T_{2} \frac{d}{d t}-T_{2} Q-T_{3} a & =\frac{d}{d t} T_{2}+h_{0} T_{2}+h_{1} T_{1}+h_{2} E-a T_{3},
\end{aligned}
$$

являющихся следствием равенства $T(d / d t-a \xi-Q)=(d / d t-a \xi-h) T$. По известному ряду (52) находим $S$ из условия $S T=D_{n}(T) g$, которое также можно записать в виде цепочки равенств. Условие совместности уравнений (51) можно представить в виде

$$
\left(D_{n}-1\right) h=\frac{d}{d t} \ln S .
$$

Отсюда ясно, что функции $h$ и $\ln S$ являются производящими функциями бесконечной серии законов сохранения. Приведем в явном виде первые три из них:

$$
\begin{gathered}
\left(D_{n}-1\right) \frac{u_{n}}{u_{n-1}}=\frac{d}{d t}\left(-\frac{1+u_{n}^{2} v_{n} v_{n+1}}{u_{n} v_{n+1}}\right), \\
\left(D_{n}-1\right) \frac{1+u_{n}^{2} v_{n} v_{n+1}}{u_{n-1} v_{n+1}}=\frac{d}{d t}\left(-\frac{u_{n+1}}{u_{n}}-\frac{1}{2}\left(u_{n} v_{n}+\frac{1}{u_{n} v_{n+1}}\right)^{2}\right), \\
\left(D_{n}-1\right) \frac{u_{n}^{4} v_{n}^{2} v_{n+1}^{2}+2 u_{n}^{2} v_{n} v_{n+1}+u_{n+1} v_{n+1}^{2} u_{n}+1}{u_{n} u_{n-1} v_{n+1}^{2}}= \\
=\frac{d}{d t}\left(-\frac{\left(1+u_{n}^{2} v_{n} v_{n+1}\right) u_{n+1}}{u_{n}^{2} v_{n+1}}-\frac{1+v_{n+1} u_{n+1}^{2} v_{n+2}}{u_{n} v_{n+2}}-\frac{1}{3}\left(u_{n} v_{n}+\frac{1}{u_{n} v_{n+1}}\right)^{3}\right) .
\end{gathered}
$$

По найденным $T, h$ и $S$ можно построить формальное асимптотическое разложение для собственной функции (решения прямой задачи рассеяния) операторов Лакса.

\section{6. ПОСТРОЕНИЕ ОБОБЩЕННОГО ИНВАРИАНТНОГО МНОГООБРАЗИЯ ДЛЯ УРАВНЕНИЯ НА КВАДРАТНОМ ГРАФЕ ПРИ ПОМОЩИ ЕГО ВЫСШЕЙ СИММЕТРИИ}

Чтобы найти обобщенное инвариантное многообразие

$$
u_{n+k, m}=F_{1}\left(n, m, u_{n, m}, u_{n+1, m}, u_{n+2, m}, \ldots, u_{n+k-1, m}\right)
$$


для уравнения на квадратном графе

$$
u_{n+1, m+1}=F\left(u_{n+1, m}, u_{n, m}, u_{n, m+1}\right),
$$

требуется решить сложное функциональное уравнение. Задача поиска обобщенного инвариантного многообразия и, следовательно, пары Лакса заметно упрощается, если известна эволюционная высшая симметрия уравнения (56) вида

$$
\frac{\partial u_{n, m}}{\partial t}=f\left(u_{n+s, m}, u_{n+s-1, m}, \ldots, u_{n-s, m}\right) .
$$

Здесь переменная $m$ зафиксирована и входит в задачу как параметр. Мы исходим из общеизвестного факта, что пары Лакса для уравнения и его симметрии имеют общий оператор. Поэтому мы предполагаем, что функция $F_{1}$ также определяет инвариантное многообразие для симметрии (57) уравнения (56). Чтобы найти поверхность (55) по уравнению (57), требуется решить переопределенную систему уже не функциональных, а дифференциальных уравнений, что существенно проще.

Поясним алгоритм поиска инвариантного многообразия через симметрию на примере дискретного уравнения, называющегося уравнением $\mathrm{dpKdV}$, или уравнением Н1 согласно классификации из работы [12]:

$$
u_{n+1, m+1}-u_{n, m}=\frac{c}{u_{n+1, m}-u_{n, m+1}} .
$$

Это уравнение допускает бесконечную иерархию высших симметрий эволюционного типа. Наиболее простая из высших симметрий имеет первый порядок:

$$
\frac{d u_{n, m}}{d t}=\frac{1}{u_{n+1, m}-u_{n-1, m}} .
$$

Линеаризации

$$
U_{n+1, m+1}=U_{n, m}-\frac{c}{q_{n, m}^{2}} U_{n+1, m}+\frac{c}{q_{n, m}^{2}} U_{n, m+1}
$$

и

$$
\frac{d U_{n, m}}{d t}=-p_{n, m}^{2}\left(U_{n+1, m}-U_{n-1, m}\right)
$$

уравнений (58) и соответственно (59) имеют в качестве коэффициентов степени переменных $p_{n, m}$ и $q_{n, m}$. Ниже нам понадобятся равенства, которые эти переменные и их сдвиги связывают между собой и с переменной $u_{n, m}$ :

$$
\begin{aligned}
p_{n, m} & =\frac{1}{u_{n+1, m}-u_{n-1, m}}, & q_{n, m} & =u_{n+1, m}-u_{n, m+1}, \\
p_{n+1, m} & =\frac{q_{n, m}}{q_{n, m} q_{n+1, m}+c}, & q_{n+1, m+1} & =q_{n, m}+\frac{c}{q_{n+1, m}}-\frac{c}{q_{n, m+1}} .
\end{aligned}
$$

Отметим, что функции $\ln p_{n, m}$ и $\ln q_{n, m}$ являются плотностями законов сохранения для уравнения (58). Действительно, легко проверить, что выполняется условие

$$
\left(D_{n}-1\right) \ln q_{n-1, m}=\left(D_{m}-1\right) \ln p_{n, m} .
$$


В работе [10] было найдено обобщенное инвариантное многообразие вида

$$
\begin{aligned}
U_{n+3, m}= & \left(-\frac{p_{n+1, m}}{p_{n+2, m}}+\frac{\lambda}{p_{n+2, m}^{2}}\right) U_{n+2, m}+ \\
& +\left(1-\frac{\lambda}{p_{n+1, m} p_{n+2, m}}\right) U_{n+1, m}+\frac{p_{n+1, m}}{p_{n+2, m}} U_{n, m}
\end{aligned}
$$

для цепочки (59), которую мы уже обсуждали в разделе 3. Как и следовало ожидать, это же уравнение определяет обобщенное инвариантное многообразие для уравнения (58), высшей симметрией которого является цепочка (59).

Чтобы полностью описать обобщенное инвариантное многообразие для уравнения на квадратном графе, необходимо вывести еще одно линейное уравнение, определяющее динамику переменной $U_{n, m}$ по аргументу $m$. Воспользуемся линеаризованным уравнением (60), представленным в виде

$$
U_{n+3, m+1}=U_{n+2, m}-\frac{c}{q_{n+2, m}^{2}} U_{n+3, m}+\frac{c}{q_{n+2, m}^{2}} U_{n+2, m+1} .
$$

В правой части полученного уравнения исключим $U_{n+3, m}$ и $U_{n+2, m+1}$ на основании (62) и соответственно (60) и тем самым приведем (63) к виду

$$
\begin{aligned}
& U_{n+3, m+1}=\left(\frac{c}{q_{n+2, m}^{2}}\left(\frac{p_{n+1, m}}{p_{n+2, m}}-\frac{\lambda}{p_{n+2, m}^{2}}\right)+1-\frac{c^{2}}{q_{n+2, m}^{2} q_{n+1, m}^{2}}\right) U_{n+2, m}+ \\
& \quad+\left(\frac{c \lambda}{p_{n+1, m} p_{n+2, m} q_{n+2, m}^{2}}-\frac{c^{3}}{q_{n+2, m}^{2} q_{n+1, m}^{2} q_{n, m}^{2}}\right) U_{n+1, m}+ \\
& \quad+\left(-\frac{c p_{n+1, m}}{p_{n+2, m} q_{n+2, m}^{2}}+\frac{c^{2}}{q_{n+2, m}^{2} q_{n+1, m}^{2}}\right) U_{n, m}+\frac{c^{3}}{q_{n+2, m}^{2} q_{n+1, m}^{2} q_{n, m}^{2}} U_{n, m+1} .
\end{aligned}
$$

Поступая аналогично, преобразуем (62) к виду

$$
\begin{aligned}
& U_{n+3, m+1}=-\frac{c}{q_{n+1, m}^{2}}\left(\frac{p_{n+1, m}}{p_{n+2, m}}-\frac{\lambda}{p_{n+2, m}^{2}}\right) U_{n+2, m}+ \\
& +\left(\left(\frac{p_{n+1, m}}{p_{n+2, m}}-\frac{\lambda}{p_{n+2, m}^{2}}\right)\left(1-\frac{c^{2}}{q_{n+1, m}^{2} q_{n, m}^{2}}\right)-\right. \\
& \left.\quad-\frac{c}{q_{n, m}^{2}}\left(1-\frac{\lambda}{p_{n+1, m+1} p_{n+2, m+1}}\right)\right) U_{n+1, m}+ \\
& +\left(\frac{c}{q_{n+1, m}^{2}}\left(-\frac{p_{n+1, m+1}}{p_{n+2, m+1}}+\frac{\lambda}{p_{n+2, m+1}^{2}}\right)+1-\frac{\lambda}{p_{n+1, m+1} p_{n+2, m+1}}\right) U_{n, m}+ \\
& +\left(\frac{c^{2}}{q_{n+1, m}^{2} q_{n, m}^{2}}\left(-\frac{p_{n+1, m+1}}{p_{n+2, m+1}}+\frac{\lambda}{p_{n+2, m+1}^{2}}\right)+\right. \\
& \left.\quad+\frac{c}{q_{n, m}^{2}}\left(1-\frac{\lambda}{p_{n+1, m+1} p_{n+2, m+1}}\right)+\frac{p_{n+1, m+1}}{p_{n+2, m+1}}\right) U_{n, m+1} .
\end{aligned}
$$

Сравнение соотношений (64) и (65) дает искомое уравнение

$$
\begin{aligned}
U_{n, m+1}= & -\frac{q_{n, m} p_{n+1, m}}{(c \lambda-1)} U_{n+2, m}+\frac{\lambda q_{n, m}-p_{n+1, m}}{p_{n+1, m}(c \lambda-1)} U_{n+1, m}+ \\
& +\frac{\left(\lambda q_{n, m}-p_{n+1, m}\right) q_{n, m}}{c \lambda-1} U_{n, m} .
\end{aligned}
$$


Ниже нам понадобятся следующие равенства, вытекающие из (62), (66):

$$
\begin{aligned}
U_{n+1, m+1}= & -\frac{c p_{n+1, m}}{(c \lambda-1) q_{n, m}} U_{n+2, m}+\frac{c \lambda q_{n+1, m}}{q_{n, m}(c \lambda-1)} U_{n+1, m}-\frac{p_{n+1, m} q_{n+1, m}}{c \lambda-1} U_{n, m}, \\
U_{n+2, m+1}= & -\frac{c\left(c \lambda-q_{n+1, m} p_{n+1, m}\right)}{q_{n+1, m}^{2}(c \lambda-1)} U_{n+2, m}+ \\
& +\frac{c \lambda-q_{n+1, m} p_{n+1, m}}{q_{n+1, m} p_{n+1, m}(c \lambda-1)} U_{n+1, m}-\frac{c p_{n+1, m}}{q_{n+1, m}(c \lambda-1)} U_{n, m} .
\end{aligned}
$$

Полагая $\varphi_{n, m}=\left(U_{n+2, m}, U_{n+1, m}, U_{n, m}\right)^{\mathrm{T}}$, перепишем скалярные уравнения $(61),(62)$ и (66) в следующей матричной форме:

$$
\varphi_{n+1, m}=f \varphi_{n, m}, \quad \varphi_{n, m+1}=g \varphi_{n, m}
$$

где

$$
\begin{aligned}
& f=\left(\begin{array}{ccc}
\frac{p_{n+1, m}}{p_{n+2, m}}+\frac{\lambda}{p_{n+2, m}^{2}} & 1-\frac{\lambda}{p_{n+1, m} p_{n+2, m}} & \frac{p_{n+1, m}}{p_{n+2, m}} \\
1 & 0 & 0 \\
0 & 1 & 0
\end{array}\right), \\
& g=\left(\begin{array}{ccc}
-\frac{c\left(c \lambda-q_{n+1, m} p_{n+1, m}\right)}{q_{n+1, m}^{2}} & \frac{c \lambda-q_{n+1, m} p_{n+1, m}}{q_{n+1, m} p_{n+1, m}} & -\frac{c p_{n+1, m}}{q_{n+1, m}} \\
-\frac{c p_{n+1, m}}{q_{n, m}} & \frac{c \lambda q_{n+1, m}}{q_{n, m}} & -p_{n+1, m} q_{n+1, m} \\
-q_{n, m} p_{n+1, m} & \frac{\lambda q_{n, m}-p_{n+1, m}}{p_{n+1, m}} & -\left(\lambda q_{n, m}-p_{n+1, m}\right) q_{n, m}
\end{array}\right) .
\end{aligned}
$$

Теорема. Система (67) совместна тогда и толъко тогда, когда выполняется уравнение (58).

Теорема доказывается непосредственным вычислением.

\section{7. ЗАКЛЮЧЕНИЕ}

В работе обсуждается задача разработки эффективного алгоритма построения пары Лакса для интегрируемого уравнения. В настоящее время наибольший интерес в теории интегрируемости вызывают сравнительно новые объекты: дискретные модели - нелинейные уравнения на квадратных графах и их симметрии - нелинейные цепочки. Несмотря на наличие интересных и глубоких результатов по классификации и интегрированию дискретных моделей, теория интегрируемых уравнений на графах далека от своего завершения. Мало изученными остаются дискретные системы экспоненциального типа - объекты, имеющие приложения в дискретной теории поля (см., например, работы [23]-[25]). Для класса интегрируемых дискретных экспоненциальных систем, связанных с аффинными алгебрами Ли, не найдены пары Лакса. Поэтому развитие методов поиска пар Лакса является актуальной задачей. Чтобы построить пару Лакса для интегрируемого уравнения, мы предлагаем следующую схему.

- Линеаризовать заданное интегрируемое уравнение.

- Найти линейное многообразие, инвариантное по отношению к линеаризованному уравнению. Пара, состоящая из линеаризованного уравнения и уравнения 
его инвариантного многообразия, определяет некоторую пару Лакса для рассматриваемого уравнения. Мы называем ее предварительной парой Лакса, так как она допускает понижение порядка.

- Найти интеграл предварительной пары Лакса и понизить ее порядок. В результате получится пара нелинейных уравнений (или систем уравнений).

- С помощью подходящей замены переменных свести нелинейную систему, полученную в предыдущем пунтке, к некоторой линейной системе, которая и будет искомой парой Лакса.

Эта схема проиллюстрирована на примерах. Для цепочки (20) по известному методу [18] построен рекурсионный оператор. Затем с помощью этого оператора найдена пара Лакса.

\section{Список литературы}

[1] В. Е. Захаров, А. Б. Шабат, "Схема интегрирования нелинейных уравнений математической физики методом обратной задачи рассеяния. I", Функи. анализ и его прил., 8:3 (1974), 43-53.

[2] В.Е. Захаров, А. Б. Шабат, "Интегрирование нелинейных уравнений математической физики методом обратной задачи рассеяния. II", Функи. анализ и его прил., 13:3 (1979), 13-22.

[3] H.D. Wahlquist, F. B. Estabrook, "Prolongation structures of nonlinear evolution equations", J. Math. Phys., 16:1 (1975), 1-7.

[4] F. W. Nijhoff, A. J. Walker, "The discrete and continuous Painlevé VI hierarchy and the Garnier system", Glasgow Math. J., 43:A (2001), 109-123.

[5] A. I. Bobenko, Yu. B. Suris, "Integrable systems on quad-graphs", Int. Math. Res. Notices, 2002:11 (2002), 573-611.

[6] F. W. Nijhoff, "Lax pair for the Adler (lattice Krichever-Novikov) system", Phys. Lett. A, 297:1-2 (2002), 49-58.

[7] Р. И. Ямилов, "О классификации дискретных уравнений", Интегрируемъе системы, ред. А. Б. Шабат, БашГУ, Уфа, 1982, 95-114.

[8] P. Xenitidis, "Integrability and symmetries of difference equations: the Adler-Bobenko-Suris case", Proceedings of IV Workshop "Group Analysis of Differential Equations and Integrable Systems" (GADEIS-IV) (Protaras, Cyprus, 26-30 October, 2008), eds. N. Ivanova, C. Sophocleous, R. Popovych, P. Damianou, A. Nikitin, University of Patras, Greece, 2008, 226-242, arXiv: 0902.3954.

[9] Ф. Ханизаде, А. В. Михайлов, Дж. П. Ванг, "Преобразования Дарбу и рекурсионные операторы для дифференциально-разностных уравнений", ТМФ, 177:3 (2013), $387-440$.

[10] I. T. Habibullin, A. R. Khakimova, M. N. Poptsova, "On a method for constructing the Lax pairs for nonlinear integrable equations", J. Phys. A: Math. Theor., 49:3 (2016), 035202, $35 \mathrm{pp}$.

[11] Р. И. Ямилов, “Классификация дискретных эволюционных уравнений", УМН, 38:6 (1983), 155-156.

[12] V.E. Adler, A.I. Bobenko, Yu.B. Suris, "Classification of integrable equations on quad-graphs. The consistency approach", Commun. Math. Phys., 233:3 (2003), 513-543.

[13] Б. И. Сулейманов, “'Квантовая’ линеаризация уравнений Пенлеве как компонента их L, A пар", Уфимск. матем. журн., 4:2 (2012), 127-135.

[14] Н.Х. Ибрагимов, А.Б. Шабат, "Уравнение Кортевега-де Фриза с групповой точки зрения", Докл. АН СССР, 244:1 (1979), 56-61.

[15] Н. Х. Ибрагимов, А.Б. Шабат, “Эволюционные уравнения с нетривиальной группой Ли-Беклунда", Функи. анализ и его прил., 14:1 (1980), 25-36. 
[16] R. Garifullin, I. Habibullin, M. Yangubaeva, "Affine and finite Lie algebras and integrable Toda field equations on discrete space-time", SIGMA, 8 (2012), 062, 33 pp.

[17] S. I. Svinolupov, R. I. Yamilov, "The multi-field Schrödinger lattices", Phys. Lett. A, 160:6 (1991), 548-552.

[18] R. I. Yamilov, "Symmetries as integrability criteria for differential difference equations", J. Phys. A: Math. Gen., 39:45 (2006), R541-R623.

[19] M. Gürses, A. Karasu, V. V. Sokolov, "On construction of recursion operators from Lax representation", J. Math. Phys., 40:12 (1999), 6473-6490.

[20] В. Г. Дринфельд, В. В. Соколов, “Алгебры Ли и уравнения типа Кортевега-де Фриза”, Итоги науки и техн. Сер. Соврем. пробл. матем. Нов. достиж., 24, ВИНИТИ, М., 1984, $81-180$.

[21] И. Т. Хабибуллин, “Дискретная система Захарова-Шабата и интегрируемые уравнения", Зап. научн. сем. ЛОМИ, 146 (1985), 137-146.

[22] A. V. Mikhailov, "Formal diagonalisation of Lax-Darboux schemes", Модел. и анализ информ. систем, 22:6 (2015), 795-817.

[23] A. Kuniba, T. Nakanishi, J. Suzuki, "T-systems and $Y$-systems in integrable systems", J. Phys. A: Math. Theor., 44:10 (2011), 103001, 146 pp., arXiv: 1010.1344.

[24] R. S. Ward, "Discrete Toda field equations", Phys. Lett. A, 199:1-2 (1995), 45-48.

[25] R. Willox, M. Hattori, "Discretisations of constrained KP hierarchies", J. Math. Sci. Univ. Tokyo, 22:3 (2015), 613-661, arXiv: 1406.5828.

Поступила в редакцию 26.04.2016, после доработки 6.02.2017 